\title{
4 layer elastic bandages were more cost effective than multilayer inelastic bandages for healing venous leg ulcers
}

Iglesias C, Nelson EA, Cullum NA, et al on behalf of the VenUS Team. VenUS I: a randomised controlled trial of two types of bandage for treating venous leg ulcers. Health Technol Assess 2004;8:iii, 1-105.

Are multilayer short stretch bandages (SSBs) more cost effective than 4 layer bandages (4LBs) for healing venous leg ulcers?

\section{METHODS}

$\square$

Design: randomised controlled trial.

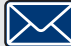

Allocation: concealed.

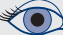

Blinding: unblinded.

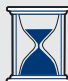

Follow up period: mean duration of 193 days.

Setting: 9 centres in the UK. The centres provided several different models of leg ulcer service, including community and district nurse led services, community leg ulcer clinics, and hospital leg ulcer clinics with community outreach.

Patients: 387 patients $\geqslant 18$ years of age (mean age 72 years, $59 \%$ women) who had an ankle brachial pressure index $\geqslant 0.8$ and venous leg ulcers for $\geqslant 1$ week that were $\geqslant 1 \mathrm{~cm}$ in length or width. Patients with diabetes were excluded.

$\mathbf{R}_{\mathbf{X}}$

Intervention: SSBs providing multilayered, inelastic compression ( $n=192$ ) or $4 \mathrm{LBs}$ providing multilayered, elastic compression $(n=195)$. The SSB system comprised a first layer of orthopaedic wool and then upper layers of cotton SSBs. 4LB systems were available as proprietary kits; kits from Profore, System 4, and Original 4LB were permitted in the trial. Both bandage systems were applied on the day of trial entry and replaced as dictated by clinical need.

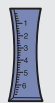

Outcomes: healing of all ulcers on the trial leg and costs of leg ulcer treatment

Patient follow up: all patients were included in the intention to treat analyses.

\section{MAIN RESULTS}

The groups did not differ for median time to healing of all ulcers on the trial leg $(126 \vee 92 \mathrm{~d}, \mathrm{p}=0.12)$. After adjusting for potential confounders (including ulceration episodes since disease onset, patient weight, area of ulceration at baseline, duration [mo current ulcer open], ankle mobility, and centre), the probability of healing of all ulcers on the trial leg was lower in the SSB group than in the 4LB group (hazard ratio $0.72,95 \%$ CI 0.57 to 0.91 ). The cost of leg ulcer University of York, York, UK. nac2@york.ac.uk

Source of funding: National Health Service Research and Development Health Technology Assessment Programme, UK. treatment in 2001 British pounds was higher in the SSB group than in the 4LB group (difference in mean cost per patient per year $£ 227$, $95 \%$ CI 16 to 448 ).

\section{CONCLUSION}

4 layer elastic compression bandages were more effective and less expensive than multilayer short stretch bandages for healing venous leg ulcers.

\section{Commentary}

Ithough we know that high compression heals venous ulcers, compelling evidence regarding the most clinically effective system of compression bandaging does not exist.' Iglesias et al attempted to address this issue by comparing multilayered inelastic bandaging with multilayered elastic bandaging over a 30 month period. The study sample was large, and the follow up period was long enough to provide confidence in the findings. Experimental and control groups were well matched and, apart from exclusion of patients with diabetes, appear to be representative of patients with venous ulcers.

Reduction of bias was achieved by allocation concealment, stratification by ulcer size and duration, intention to treat analysis, and confirmation of healing by remote observer blinded to allocation. Clinical applicability is an important concept that helps to assure clinicians that the results can be applied to their clinical settings. Iglesias et al attempted to increase generalisability by conducting the study in various clinical settings including community, district nurse services, and hospital based clinics.

Key findings of this study were that $4 \mathrm{LBs}$ increased the probability of venous leg ulcer healing more than SSBs and reduced the cost of treatment. Clinical outcomes of studies involving application of compression bandages can be influenced by the skill level of the nurses applying the bandages. This is a result of the learning curve associated with applying a new type of compression bandage. In such situations, the outcome is usually in favour of the established bandaging system, ${ }^{2}$ which in this case is the $4 \mathrm{LB}$ system. Because the authors did not measure competence of bandage application at baseline, the influence of this phenomenon should be noted when considering these results.

This study provides clinicians with information about the more favourable clinical and economic outcomes of $4 \mathrm{LBs}$ compared with SSBs in the treatment of venous leg ulcers. For clinicians currently using SSBs, staff training costs should be considered in the decision to change protocols.

Julie Betts, RGON, AdDipN Health Waikato Hamilton, New Zealand

1 Cullum N, Nelson EA, Fletcher AW, et al. Compression for venous leg ulcers. Cochrane Database Syst Rev 2001;(2):CD000265.

2 Nelson EA, Ruckley CV, Barbenel JC. Improvements in bandaging technique following training. J Wound Care 1995;4:181-4. 